LEARNING DISORDERS

ASPARTAME AND LEARNING AND BEHAVIOR

The effects of aspartame on learning, behavior, and mood of 910-year-old normal children were examined in the Department of Nutritional Sciences, University of Toronto, Ontario, Canada. Measures of associative learning, arithmetic calculation, activity level, social interaction and mood were unaffected by treatment with Kool-Aid containing $1.76 \mathrm{gram} / \mathrm{kg}$ of carbohydrate (polycose) plus either aspartame $(34 \mathrm{mg} / \mathrm{kg})$ or the equivalent sweetness as sodium cyclamate and amino acids as alanine. In a second experiment in which children received a drink of cold, unsweetened strawberry Kool-Aid containing either 1.75 gram $/ \mathrm{kg}$ of sucrose or $9.7 \mathrm{mg} / \mathrm{kg}$ of aspartame, the frequency of minor and gross motor behaviors was significantly less after the consumption of sucrose than after aspartame treatment. (Saravis S et al. Aspartame: effects on learning, behavior and mood. Pediatrics July $1990 ; 86: 75-83$ ).

COMMENT: The authors concluded that the effects of aspartame on short-term behavior were more likely due to an "absence of metabolic consequences of providing sweetness rather than to neurochemical consequences related to its amino acid composition". The observed reduction in activity following sucrose ingestion is in agreement with some previous reports and adds to the controversy concerning hyperactivity and sugar. The tests in the above study that failed to show significant effects of aspartame included the Conditional Associative Learning Task, the Canadian Tests of Basic Skills, the Children's Depression Inventory and the State-Trait Anxiety Inventory for Children. The minor and gross motor behaviors which showed significant improvement with sucrose included the actameter measure, a modified self-winding wrist watch, and a video taped observation of behavior. The children in this study were normal and different responses may occur in patients with ADHD.

HANDEDNESS, STUTTERING AND ALLERGIES

The relationship of left-handedness to allergic disorders and stuttering, using epidemiological data of two French samples, is reported from L'Institut National de la Sante et de la Recherche Medicale, Villejuif, France. A higher frequency of stuttering, but not of allergic disorders, occured in left-handers. Extreme right-handedness was significantly associated with a lower frequency of allergic disorders. The Geschwind-Galaburda theory of cerebral dominance was not supported by these findings. Any significant association of allergic disorders with handedness disappeared after the exclusion of the extreme right-handers. On the contrary, stuttering was associated with left-handedness even after exclusion of extreme handedness. (Dellatolas $\mathrm{G}$ et al. An epidemiological reconsideration of the Geschwind-Galaburda theory of cerebral lateralization. Arch Neurol July $1990 ; 47: 778-782$ ). 
COMMENT The Geschwind-Galaburda theory of cerebral daminance invokes in utero testosterone-induced-immune disorders and left-handedness by effects on the thymus and the brain. Their clinical observations showed a higher frequency of immune diseases, migraine, and developmental learning disorders in left-handed individuals and their families.

ALEXIA, AGRAPHIA, AND FRONTAL LOBE DAMAGE

The case history of a right-handed woman who developed severe and stable alexia and agraphia following a circumscribed surgical lesion in the premotor cortex is reported from the Department of Neurology, Division of Behavioral Neurology and Cognitive Neuroscience, University of Iowa College of Medicine, Iowa City, Iowa. The lesion was above Broca's area in Exner's area. Her visual perception, intellect, memory, oral spelling, and drawing were normal and she was not aphasic or hemiparetic. She was unable to read sentences and her reading of single words and letters was severely impaired. She could not write recognizable letters or words. By contrast she was able to write numbers and perform written calculations without difficulty. These dissociations of function provide evidence of specificity of cognitive and neural representation. (Anderson SW, Demasio AR, Demasio H. Troubled letters but not numbers. Domain specific cognitive impairments following focal damage in frontal cortex. Brain June 1990; 113: 749-766).

COMMENT The isolated simultaneous occurrence of alexia and agraphia is rare. In this case the anatomical lesion within the left frontal lobe was unusually circumscribed. The sector of association cortex in the frontal lobe, known as Exner's area, appears to be related to the ability to read and write. The pathology of the small lesion removed surgically was a single metastasis from an adenocarcinoma of the lung. Neurological evaluation was normal except for the cognitive defect described.

The neuroanatomical basis of developmental dyslexia has been debated since this hypothesis was proposed by Orton in 1937. Patterns of task related slow-brain potentials have been investigated in six dyslexic youths by Landwehrmeyer B et al (Arch Neurol July 1990; 47:791-797). Whereas control subjects revealed greater left hemisphere negativity during linguistic tasks, the reverse was found with dyslexics. The authors, working at the Neurologische Universitatsklinik, Freiburg, West Germany, concluded that dyslexia is associated with changes in the lateral distribution of cortical activity during certain types of language processing.

\section{FRONTAL LOBE FUNCTION AND ATTENTION DEFICITS}

The results of a psychological test battery administered to 54 clinic referred children aged 8 to 12 years with attention deficit disorders are reported from the Georgia Children's Center and the Department of Psychology, University of Oregon, Eugene, Oregon. 